\title{
Symmetry of Solutions for a Fractional System
}

\author{
Yan Li Pei Ma*
}

August 7, 2018

\begin{abstract}
We consider the following equations:

$$
\begin{cases}(-\triangle)^{\alpha / 2} u(x)=f(v(x)), & \\ (-\triangle)^{\beta / 2} v(x)=g(u(x)), & x \in R^{n} \\ u, v \geq 0, & x \in R^{n}\end{cases}
$$

for continuous $f, g$ and $\alpha, \beta \in(0,2)$. Under some natural assumptions on $f$ and $g$, by applying the method of moving planes directly to the system, we obtain symmetry on non-negative solutions without any decay assumption on the solutions at infinity.
\end{abstract}

Key words The fractional Laplacian, narrow region principle, decay at infinity, method of moving planes, radial symmetry.

\section{Introduction}

The famous Lane-Emden equation

$$
\left\{\begin{array}{l}
-\triangle u=u^{p}, \\
u>0,
\end{array} \quad x \in R^{n},\right.
$$

has been the central part in the progression of nonlinear analysis in the past few decades. Such fundamental results as the critical point theory (also

\footnotetext{
${ }^{*}$ Corresponding author.
} 
known as the mountain pass theory), the a priori estimates, the eigenfunction theory and the Liouville-type theorems have been obtained (please see [1]-[5] and the references therein).

Naturally, one would want to know if these results are valid for the LaneEmden system:

$$
\left\{\begin{array}{l}
-\triangle u(x)=v^{q}(x), \\
-\triangle v(x)=u^{p}(x), \quad x \in R^{n} \\
u, v>0
\end{array}\right.
$$

with $p, q>0$. Among which, the problem of existence and nonexistence (known as the Liouville theorem) of solutions has attracted wide attention, but have not yet been fully answered. The famous Lane-Emden conjecture states that

Problem (2) has no classical solutions in the subcritical case $\frac{1}{p+1}+\frac{1}{q+1}>$ $\frac{n-2}{n}$.

The conjecture was validated by Mitidieri [6] for radial solutions. In the critical case $\frac{1}{p+1}+\frac{1}{q+1}=\frac{n-2}{n}$ and the supercritical case $\frac{1}{p+1}+\frac{1}{q+1}<\frac{n-2}{n}$, it has been proved in [7] and [6] that (2) has bounded radial classical solutions.

In $R^{3}$, Serrin and Zou [7] confirmed the conjecture on condition that $(u, v)$ are bounded by polynomials at infinity. A decade later, Poláčik, Quittner and Souplet [8] removed the growth assumption and obtained same results. Recently, still without growth restrictions at infinity, Souplet [9] proved the full conjecture in $R^{4}$ and in a particular category in dimensions higher than 4 . Partial results in higher dimensions were also obtained in certain subregions by Felmer and Figueiredo [10, Chen and Li [11], Busca and Manásevich [12. and Lin [13]. The Lane-Emden conjecture was also considered for higher order elliptic systems:

$$
\left\{\begin{array}{l}
(-\triangle)^{m} u(x)=v^{q}(x), \\
(-\triangle)^{m} v(x)=u^{p}(x), \quad x \in R^{n}, \\
u, v>0
\end{array}\right.
$$

where $p, q>0$ and $n>1, m \in N$. Partial results were obtained by Mitidieri[6], Yan [14], Arthur, Yan and Zhao [15].

The counterpart of the Lane-Emden equation in the fractional Laplacian setting has also received a lot of attention.

The fractional Laplacian $(-\triangle)^{\alpha / 2}$ is a nonlocal pseudo-differential oper- 
ator defined as

$$
\begin{aligned}
(-\triangle)^{\alpha / 2} u(x) & =C_{n, \alpha} P V \int_{\mathbb{R}^{n}} \frac{u(x)-u(z)}{|x-z|^{n+\alpha}} d z \\
& =C_{n, \alpha} \lim _{\epsilon \rightarrow 0} \int_{\mathbb{R}^{n} \backslash B_{\epsilon}(x)} \frac{u(x)-u(z)}{|x-z|^{n+\alpha}} d z,
\end{aligned}
$$

for $\alpha \in(0,2)$. Here PV stands for the Cauchy principle value.

Let

$$
L_{\alpha}=\left\{u: \mathbb{R}^{n} \rightarrow \mathbb{R} \mid \int_{\mathbb{R}^{n}} \frac{|u(x)|}{1+|x|^{n+\alpha}} d x<\infty\right\} .
$$

Then it's easy to see that for $u \in L_{\alpha} \cap C_{l o c}^{1,1}\left(R^{n}\right)$, the integral on the right hand side of (44) is well defined. In this paper, we consider the fractional Laplacian in this space.

The fractional Laplacian has been used to describe problems emerging from multiple fields. For example, in the Lévy process, it has been studied as the the infinitesimal generator from a perspective of the probability (see [16], [17]). Its nature of non-locality also fits into various research subjects as phase transitions, optimization, flame propagation, finance, and so on. Readers who are interested please see [18], [19], [20], 21], [22] and the references therein.

In [23], the authors considered

$$
\begin{cases}(-\triangle)^{\alpha / 2} u=u^{p}, & x \in R_{+}^{n}, \\ u \equiv 0, & x \notin R_{+}^{n} .\end{cases}
$$

They first proved that (5) is equivalent to an integral equation

$$
u(x)=\int_{R_{+}^{n}} G(x, y) u^{p}(y) d y,
$$

where $G(x, y)$ is the Green's function for $(-\triangle)^{\alpha / 2}$ in $R_{+}^{n}$. Then by applying the method of moving planes in integral forms, they proved the non-existence of positive solutions for $p \leq \frac{n+\alpha}{n-\alpha}$ without assumptions on the growth of the solutions. Using the same method, the authors in 24] obtained radial symmetry of positive solutions for a system:

$$
\begin{cases}(-\triangle)^{\alpha / 2} u_{i}=f_{i}\left(u_{1}, u_{2}, \cdots, u_{m}\right), & i=1,2, \cdots, m, \\ u_{i}>0, & x \in R^{n},\end{cases}
$$


by investigating its corresponding integral system:

$$
\begin{cases}u_{i}=\int_{R^{n}} \frac{C_{n, \alpha}}{|x-y|^{n-\alpha}} f_{i}(u(y)), & i=1,2, \cdots, m, \\ u_{i}>0, & x \in R^{n} .\end{cases}
$$

For more details on the method of moving planes in integral forms, please see [25]-29]. In [30, the authors derived existence and uniqueness of positive viscosity solutions on a smooth bounded domain $\Omega \subseteq R^{n}$ :

$$
\begin{cases}(-\triangle)^{\alpha / 2} u(x)=v^{q}(x), & \\ (-\triangle)^{\alpha / 2} v(x)=u^{p}(x), & x \in \Omega, \\ u=v=0, & x \notin \Omega,\end{cases}
$$

when $p q \neq 1, p, q>0$ in the supercritical case $\frac{1}{p+1}+\frac{1}{q+1}>\frac{n+\alpha}{n}$. Quite recently, Chen, Li and Li [31] developed a direct method of moving planes for the fractional Laplacian. With this, they derived symmetry and nonexistence for:

$$
\left\{\begin{array}{l}
(-\triangle)^{\alpha / 2} u(x)=u^{p}(x) \\
u>0
\end{array}\right.
$$

in $R^{n}$ and $R_{+}^{n}$. In [32], Quaas and Xia proved a non-existence result for positive viscosity solutions for:

$$
\begin{cases}(-\triangle)^{\alpha / 2} u(x)=v^{q}(x), & \\ (-\triangle)^{\alpha / 2} v(x)=u^{p}(x), & x \in R_{+}^{n}, \\ u=v=0, & x \notin R_{+}^{n},\end{cases}
$$

with $1<p, q<\frac{n+2 \alpha}{n-2 \alpha}$.

So far, quite some results have been accumulated about the fractional system involving the same order of operators. For example, in [14], Yu considered systems of the same fractional order with quite general nonlinearities. Using the method of moving planes in integral forms, among which, the author obtained symmetry of positive solutions. Such results on the system have also been proved in [33] and 34]. Till now, few have been presented dealing with equations involving different orders. In this paper, we introduce a new idea - the iteration method - to deal with such problems.

We consider the following system:

$$
\left\{\begin{array}{l}
(-\triangle)^{\alpha / 2} u(x)=f(v(x)), \\
(-\triangle)^{\beta / 2} v(x)=g(u(x)), \quad x \in R^{n} \\
u, v \geq 0
\end{array}\right.
$$


with $\alpha, \beta>0$.

First, we use the iteration method to establish the maximum principles for the system.

Let $T_{\lambda}$ be a hyperplane in $\mathbb{R}^{n}$. Without loss of generality, we may assume that

$$
T_{\lambda}=\left\{x=\left(x_{1}, x^{\prime}\right) \in \mathbb{R}^{n} \mid x_{1}=\lambda, \lambda \in \mathbb{R}\right\} .
$$

Let

$$
x^{\lambda}=\left(2 \lambda-x_{1}, x_{2}, \ldots, x_{n}\right), \quad \Sigma_{\lambda}=\left\{x \in \mathbb{R}^{n} \mid x_{1}<\lambda\right\} .
$$

Theorem 1 (Decay at Infinity) For $0<\alpha, \beta<2$, assume that $U \in$ $L_{\alpha}\left(R^{n}\right) \cap C_{\text {loc }}^{1,1}(\Omega), V \in L_{\beta}\left(R^{n}\right) \cap C_{\text {loc }}^{1,1}(\Omega)$, and $U, V$ are lower semi-continuous on $\bar{\Omega}$. If

$$
\begin{cases}(-\triangle)^{\alpha / 2} U(x)+c_{1}(x) V(x) \geq 0, & \\ (-\triangle)^{\beta / 2} V(x)+c_{2}(x) U(x) \geq 0, & x \in \Omega, \\ U(x), V(x) \geq 0, & x \in \Sigma_{\lambda} \backslash \Omega, \\ U\left(x^{\lambda}\right)=-U(x), & x \in \Sigma_{\lambda},\end{cases}
$$

with

$$
c_{1}(x) \sim o\left(\frac{1}{|x|^{\alpha}}\right), \quad c_{2}(x) \sim o\left(\frac{1}{|x|^{\beta}}\right), \quad \text { for }|x| \text { large },
$$

and

$$
c_{i}(x)<0, \quad i=1,2,
$$

then there exists a constant $R>0$ (depending on $c_{i}(x)$, but is independent of $U, V$ ) such that if

$$
U(\tilde{x})=\min _{\Omega} U(x)<0, \quad V(\bar{x})=\min _{\Omega} V(x)<0,
$$

then

$$
|\tilde{x}|,|\bar{x}| \leq R
$$

Theorem 2 (Narrow Region Principle) Let $\Omega$ be a bounded narrow region in $\Sigma_{\lambda}$, such that it is contained in $\left\{x \mid \lambda-l<x_{1}<\lambda\right\}$ with small $l$. For $0<\alpha, \beta<2$, assume that $U \in L_{\alpha}\left(R^{n}\right) \cap C_{l o c}^{1,1}(\Omega), V \in L_{\beta}\left(R^{n}\right) \cap C_{l o c}^{1,1}(\Omega)$, and $U, V$ are lower semi-continuous on $\bar{\Omega}$. If $c_{i}(x)<0, i=1,2$, are bounded 
from below in $\Omega$ and

$$
\begin{cases}(-\triangle)^{\alpha / 2} U(x)+c_{1}(x) V(x) \geq 0, & \\ (-\triangle)^{\beta / 2} V(x)+c_{2}(x) U(x) \geq 0, & \text { in } \Omega, \\ U(x), V(x) \geq 0, & \text { in } \Sigma_{\lambda} \backslash \Omega \\ U\left(x^{\lambda}\right)=-U(x), & \text { in } \Sigma_{\lambda}, \\ V\left(x^{\lambda}\right)=-V(x), & \end{cases}
$$

then for sufficiently small $l$, we have

$$
U(x), V(x) \geq 0 \text { in } \Omega .
$$

If $\Omega$ is unbounded, the conclusion still holds under the condition that

$$
\varliminf_{|x| \rightarrow \infty} U(x), V(x) \geq 0 .
$$

Further, if $U(x)$ or $V(x)$ attains 0 somewhere in $\Sigma_{\lambda}$, then

$$
U(x)=V(x) \equiv 0, \quad x \in R^{n} .
$$

With the above theorem, we use a direct method of moving planes for the fractional Laplacians [31] to show that

Theorem 3 Assume that for $r \geq 0, f, g$ are nonnegative continuous functions satisfying:

(a) $f(r)$ and $g(r)$ are non-decreasing about $r$;

(b) $\frac{f(r)}{r^{p}}, \frac{g(r)}{r^{q}}$ are bounded near $r=0$ and non-increasing with $p=\frac{n+\alpha}{n-\beta}$ and $q=\frac{n+\beta}{n-\alpha}$.

If $u$ and $v$ are nonnegative solutions for (7), then

- either $u$ and $v$ are constant,

- or $f(v)=C_{1} v^{\frac{n+\alpha}{n-\beta}}$ and $g(u)=C_{2} u^{\frac{n+\beta}{n-\alpha}}$.

In particular, from Theorem 3, we have

Theorem 4 Assume $f$ and $g$ satisfy the conditions in Theorem [3. If $u$ and $v$ are nonnegative solutions for (7), then when $\alpha=\beta$,

- either $u$ and $v$ are constant, 
- $\operatorname{or} u(x)=C_{1}\left(\frac{c}{c^{2}+\left|x-x_{0}\right|^{2}}\right)^{\frac{n-\alpha}{2}}$ and $v(x)=C_{2}\left(\frac{c}{c^{2}+\left|x-x_{0}\right|^{2}}\right)^{\frac{n-\alpha}{2}}$

Remark 1.1 The form of the radial solutions were first obtained in [35] where the authors classified the positive solutions of

$$
\left\{\begin{array}{l}
u(x)=\int_{R^{n}} \frac{v^{\frac{n+\alpha}{n-\alpha}}(y)}{|x-y|^{n-\alpha}} d y \\
v(x)=\int_{R^{n}} \frac{u^{n+\alpha} \frac{\alpha}{n-\alpha}(y)}{|x-y|^{n-\alpha}} d y
\end{array}\right.
$$

More than a decade later, the author in [14] used results in [35] and obtained the same explicit expressions for

$$
\left\{\begin{array}{l}
u(x)=\int_{R^{n}} \frac{f(v)(y)}{|x-y|^{n-\alpha}} d y \\
v(x)=\int_{R^{n}} \frac{g(u)(y)}{|x-y|^{n-\alpha}} d y
\end{array}\right.
$$

The paper is organized as follows. In Section 2, we verify Theorem 1 and 2. To better illustrate the idea, we first prove Theorem 3 under assumptions on the decay rate of solutions at infinity in Section 3. Then we complete the proof of Theorem 3 in Section 4. In Section 5, we briefly prove Theorem 4 . Throughout the paper, we denote $C, C_{i}, i \in N$ as positive constants whose values may vary from line to line.

\section{Proof of the Maximum Principles}

Our proof of the maximum principles for the system is inspired by that in [31]. Let $\tilde{\Sigma}_{\lambda}=\left\{x \mid x^{\lambda} \in \Sigma_{\lambda}\right\}$. 
Proof of Theorem 1. By (41), we have

$$
\begin{aligned}
(-\triangle)^{\alpha / 2} U(\tilde{x}) & =C_{n, \alpha} P V \int_{\mathbb{R}^{n}} \frac{U(\tilde{x})-U(y)}{|\tilde{x}-y|^{n+\alpha}} d y \\
& =C_{n, \alpha} P V\left\{\int_{\Sigma_{\lambda}} \frac{U(\tilde{x})-U(y)}{|\tilde{x}-y|^{n+\alpha}} d y+\int_{R^{n} \backslash \Sigma_{\lambda}} \frac{U(\tilde{x})-U(y)}{|\tilde{x}-y|^{n+\alpha}} d y\right\} \\
& =C_{n, \alpha} P V\left\{\int_{\Sigma_{\lambda}} \frac{U(\tilde{x})-U(y)}{|\tilde{x}-y|^{n+\alpha}} d y+\int_{\Sigma_{\lambda}} \frac{U(\tilde{x})-U\left(y^{\lambda}\right)}{\left|\tilde{x}-y^{\lambda}\right|^{n+\alpha}} d y\right\} \\
& =C_{n, \alpha} P V\left\{\int_{\Sigma_{\lambda}} \frac{U(\tilde{x})-U(y)}{|\tilde{x}-y|^{n+\alpha}} d y+\int_{\Sigma_{\lambda}} \frac{U(\tilde{x})+U(y)}{\left|\tilde{x}-y^{\lambda}\right|^{n+\alpha}} d y\right\} \\
& \leq C_{n, \alpha} \int_{\Sigma_{\lambda}}\left\{\frac{U(\tilde{x})-U(y)}{\left.\left|\tilde{x}-y^{\lambda}\right|^{n+\alpha}+\frac{U(\tilde{x})+U(y)}{\left|\tilde{x}-y^{\lambda}\right|^{n+\alpha}}\right\} d y}\right. \\
& =C_{n, \alpha} \int_{\Sigma_{\lambda}} \frac{2 U(\tilde{x})}{\left|\tilde{x}-y^{\lambda}\right|^{n+\alpha}} d y .
\end{aligned}
$$

For each fixed $\lambda$, for $\tilde{x} \in \Sigma_{\lambda}$ and $|\tilde{x}|$ sufficiently large, there exists a $C>0$ such that

$$
\int_{\Sigma_{\lambda}} \frac{1}{\left|\tilde{x}-y^{\lambda}\right|^{n+\alpha}} d y \geq \int_{B_{3|\tilde{x}|}(\tilde{x}) \backslash B_{2|\tilde{x}|}(\tilde{x})} \frac{1}{|\tilde{x}-y|^{n+\alpha}} d y \sim \frac{C}{|\tilde{x}|^{\alpha}} .
$$

Hence

$$
(-\triangle)^{\alpha / 2} U(\tilde{x}) \leq \frac{C U(\tilde{x})}{|\tilde{x}|^{\alpha}}<0 .
$$

Together with (8), it's easy to deduce that

$$
V(\tilde{x})<0
$$

and

$$
U(\tilde{x}) \geq-C c_{1}(\tilde{x})|\tilde{x}|^{\alpha} V(\tilde{x}) .
$$

From (16), we know that there exists a $\bar{x}$ such that

$$
V(\bar{x})=\min _{\Omega} V(x)<0 .
$$

Similar to (17), we can derive that

$$
(-\triangle)^{\beta / 2} V(\bar{x}) \leq \frac{C V(\bar{x})}{|\bar{x}|^{\beta}}<0 .
$$


Combining (8) and (17), for $\lambda$ sufficiently negative, we have

$$
\begin{aligned}
0 & \leq(-\triangle)^{\beta / 2} V(\bar{x})+c_{2}(\bar{x}) U(\bar{x}) \\
& \leq \frac{C V(\bar{x})}{|\bar{x}|^{\beta}}+c_{2}(\bar{x}) U(\tilde{x}) \\
& \leq C\left(\frac{V(\bar{x})}{|\bar{x}|^{\beta}}-c_{2}(\bar{x}) c_{1}(\tilde{x})|\tilde{x}|^{\alpha} V(\tilde{x})\right) \\
& \leq C\left(\frac{V(\bar{x})}{|\bar{x}|^{\beta}}-c_{2}(\bar{x}) c_{1}(\tilde{x})|\tilde{x}|^{\alpha} V(\bar{x})\right) \\
& \leq \frac{C V(\bar{x})}{|\bar{x}|^{\beta}}\left(1-c_{1}(\tilde{x})|\tilde{x}|^{\alpha} c_{2}(\bar{x})|\bar{x}|^{\beta}\right) \\
& <0 .
\end{aligned}
$$

The last inequality follows from assumptions (9). This contradiction shows that (10) must be true. Through an entirely identical argument one can prove the rest of (10).

This completes the proof.

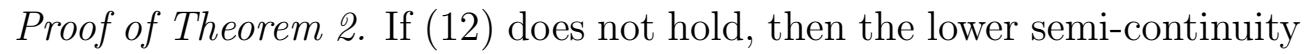
of $U$ on $\bar{\Omega}$ guarantees that there exists some $\tilde{x} \in \bar{\Omega}$ such that

$$
U(\tilde{x})=\min _{\bar{\Omega}} U<0 .
$$

And one can further deduce from condition (11) that $\tilde{x}$ is in the interior of $\Omega$.

By (4), we have

$$
\begin{aligned}
(-\triangle)^{\alpha / 2} U(\tilde{x}) & =C_{n, \alpha} P V \int_{\mathbb{R}^{n}} \frac{U(\tilde{x})-U(y)}{|\tilde{x}-y|^{n+\alpha}} d y \\
& \leq C_{n, \alpha} \int_{\Sigma_{\lambda}} \frac{2 U(\tilde{x})}{\left|\tilde{x}-y^{\lambda}\right|^{n+\alpha}} d y .
\end{aligned}
$$

Let $D=B_{2 l}(\tilde{x}) \cap \tilde{\Sigma}_{\lambda}$. Then we have

$$
\begin{aligned}
\int_{\Sigma_{\lambda}} \frac{1}{\left|\tilde{x}-y^{\lambda}\right|^{n+\alpha}} d y & \geq \int_{D} \frac{1}{|\tilde{x}-y|^{n+\alpha}} d y \\
& \geq \frac{1}{10} \int_{B_{2 l}(\tilde{x})} \frac{1}{|\tilde{x}-y|^{n+\alpha}} d y \\
& \geq \frac{C}{l^{\alpha}} .
\end{aligned}
$$


Thus,

$$
(-\triangle)^{\alpha / 2} U(\tilde{x}) \leq \frac{C U(\tilde{x})}{l^{\alpha}}<0
$$

Together with (11), we have

$$
U(\tilde{x}) \geq-c_{1}(\tilde{x}) l^{\alpha} V(\tilde{x}) .
$$

From (20), we know that there exists a $\bar{x}$ such that

$$
V(\bar{x})=\min _{\Omega} V(x)<0 .
$$

Similar to (19), we can derive that

$$
(-\triangle)^{\beta / 2} V(\bar{x}) \leq \frac{C V(\bar{x})}{l^{\beta}}<0 .
$$

Together (20), for $l$ sufficiently negative, we have

$$
\begin{aligned}
0 & \leq(-\triangle)^{\beta / 2} V(\bar{x})+c_{2}(\bar{x}) U(\bar{x}) \\
& \leq \frac{C V(\bar{x})}{l^{\beta}}+c_{2}(\bar{x}) U(\tilde{x}) \\
& \leq C\left(\frac{V(\bar{x})}{l^{\beta}}-c_{2}(\bar{x}) c_{1}(\tilde{x}) l^{\alpha} V(\tilde{x})\right) \\
& \leq C\left(\frac{V(\bar{x})}{l^{\beta}}-c_{2}(\bar{x}) c_{1}(\tilde{x}) l^{\alpha} V(\bar{x})\right) \\
& \leq \frac{C V(\bar{x})}{l^{\beta}}\left(1-c_{1}(\tilde{x}) c_{2}(\bar{x}) l^{\alpha+\beta}\right) \\
& <0 .
\end{aligned}
$$

This contradiction shows that (12) must be true.

To prove (13), we suppose that there exists $\eta \in \Omega$ such that

$$
V(\eta)=0 .
$$

Then

$$
\begin{aligned}
& (-\triangle)^{\alpha / 2} V(\eta) \\
= & C_{n, \alpha} P V \int_{\mathbb{R}^{n}} \frac{-V(y)}{|\eta-y|^{n+\alpha}} d y \\
= & C_{n, \alpha} P V \int_{\Sigma_{\lambda}} \frac{-V(y)}{|\eta-y|^{n+\alpha}} d y+\int_{\Sigma_{\lambda}} \frac{-V\left(y^{\lambda}\right)}{\left|\eta-y^{\lambda}\right|^{n+\alpha}} d y \\
= & C_{n, \alpha} P V \int_{\Sigma_{\lambda}}\left(\frac{1}{\left|x^{0}-y^{\lambda}\right|^{n+\alpha}}-\frac{1}{\left|x^{0}-y\right|^{n+\alpha}}\right) V(y) d y .
\end{aligned}
$$


If $V(x) \not \equiv 0$, then (21) implies that

$$
(-\triangle)^{\alpha / 2} V(\eta)<0 .
$$

Together with (11), it shows that

$$
U(\eta)<0 .
$$

This is a contradiction with (12). Hence $V(x)$ must be identically 0 in $\Sigma_{\lambda}$. Since

$$
V\left(x^{\lambda}\right)=-V(x), x \in \Sigma_{\lambda},
$$

it shows that

$$
V(x) \equiv 0, \quad x \in R^{n} .
$$

Again from (11), we know that

$$
U(x) \leq 0, \quad x \in \Sigma_{\lambda} .
$$

Since we already know that

$$
U(x) \geq 0, x \in \Sigma_{\lambda},
$$

it must hold that

$$
U(x)=0, x \in \Sigma_{\lambda} .
$$

Together with $U\left(x^{\lambda}\right)=U(x)$, we arrive at

$$
U(x) \equiv 0, x \in R^{n} .
$$

Similarly, one can show that if $U(x)$ attains 0 at one point in $\Sigma_{\lambda}$, then both $U(x)$ and $V(x)$ are identically 0 in $R^{n}$.

This completes the proof.

\section{Radial Symmetry under Decay-rate Assump- tion}

To carry out the method of moving planes, we need to know the behavior of the solutions at infinity. We first prove Theorem 3 in an easier case by 
assuming that for $|x|$ large, $b(p-1) \geq \beta$ and $a(q-1)>\alpha($ or $b(p-1)>\beta$ and $a(q-1) \geq \alpha)$,

$$
u(x) \sim \frac{1}{|x|^{a}}, \quad v(x) \sim \frac{1}{|x|^{b}},
$$

In the next section, one can see that even without such growth assumption, Theorem 3 still holds.

Choose an arbitrary direction for the $x_{1}$-axis. Let

$$
\begin{gathered}
u_{\lambda}(x)=u\left(x^{\lambda}\right), \\
\tilde{U}_{\lambda}(x)=u_{\lambda}(x)-u(x), \quad \tilde{V}_{\lambda}(x)=v_{\lambda}(x)-v(x) .
\end{gathered}
$$

Step 1. Start moving the plane $T_{\lambda}$ from $-\infty$ to the right along the $x_{1}$-axis.

We will show that for $\lambda$ sufficiently negative,

$$
\tilde{U}_{\lambda}(x), \tilde{V}_{\lambda}(x) \geq 0, \quad x \in \Sigma_{\lambda} \backslash\left\{0^{\lambda}\right\} .
$$

For fixed $\lambda$, by the decay rate, we know that

$$
u_{\lambda}(x) \rightarrow 0, \quad|x| \rightarrow \infty .
$$

As $\lambda \rightarrow-\infty$, for $x \in \Sigma_{\lambda}$,

$$
u(x) \rightarrow 0 .
$$

Thus for $x \in \Sigma_{\lambda}$,

$$
\tilde{U}_{\lambda}(x) \rightarrow 0, \quad \lambda \rightarrow-\infty .
$$

Similarly, one can show that for $x \in \Sigma_{\lambda}$,

$$
\tilde{V}_{\lambda}(x) \rightarrow 0, \quad \lambda \rightarrow-\infty .
$$

If

$$
\Sigma_{\tilde{U}_{\lambda}}^{-}=\left\{x \in \Sigma_{\lambda} \mid \tilde{U}_{\lambda}(x)<0\right\} \neq \emptyset,
$$

then by (221) and Lemma 6.1, we know that there must exist some $\tilde{x} \in \Sigma_{\lambda}$ such that

$$
\tilde{U}_{\lambda}(\tilde{x})=\min _{\Sigma_{\lambda}} \tilde{U}_{\lambda}<0 .
$$

On one hand, by (15),

$$
(-\triangle)^{\alpha / 2} \tilde{U}_{\lambda}(\tilde{x}) \leq \frac{C \tilde{U}_{\lambda}(\tilde{x})}{|\tilde{x}|^{\alpha}}<0 .
$$


On the other hand, by (7),

$$
(-\triangle)^{\alpha / 2} \tilde{U}_{\lambda}(\tilde{x})=f\left(v_{\lambda}(\tilde{x})\right)-f(v(\tilde{x})) .
$$

Therefore, by the monotonicity of $f$, we have

$$
\tilde{V}_{\lambda}(\tilde{x})<0 .
$$

This implies that there exists some $\bar{x} \in \Sigma_{\lambda}$ such that

$$
\tilde{V}_{\lambda}(\bar{x})=\min _{\Sigma_{\lambda}} \tilde{V}_{\lambda}<0 .
$$

By the mean value theorem, we have

$$
\begin{aligned}
& (-\triangle)^{\alpha / 2} \tilde{U}_{\lambda}(\tilde{x}) \\
= & f\left(v_{\lambda}(\tilde{x})\right)-f(v(\tilde{x})) \\
= & \frac{f\left(v_{\lambda}(\tilde{x})\right)}{v_{\lambda}^{p}(\tilde{x})} v_{\lambda}^{p}(\tilde{x})-\frac{f(v(\tilde{x}))}{v^{p}(\tilde{x})} v^{p}(\tilde{x}) \\
\geq & \frac{f(v(\tilde{x}))}{v^{p}(\tilde{x})}\left[v_{\lambda}^{p}(\tilde{x})-v^{p}(\tilde{x})\right] \\
= & \frac{f(v(\tilde{x}))}{v^{p}(\tilde{x})} p \xi^{p-1}(\tilde{x}) \tilde{V}_{\lambda}(\tilde{x}) \quad \xi \in\left[v_{\lambda}(\tilde{x}), v(\tilde{x})\right] \\
\geq & \frac{f(v(\tilde{x}))}{v^{p}(\tilde{x})} p v^{p-1}(\tilde{x}) \tilde{V}_{\lambda}(\tilde{x}) .
\end{aligned}
$$

Through a similar argument, one can show that

$$
\tilde{U}_{\lambda}(\bar{x})<0
$$

and

$$
(-\triangle)^{\beta / 2} \tilde{V}_{\lambda}(\bar{x}) \geq \frac{g(u(\bar{x}))}{u^{q}(\bar{x})} q u^{q-1}(\bar{x}) \tilde{U}_{\lambda}(\bar{x}),
$$

Let

$$
c_{1}(x)=-\frac{f(v(\tilde{x}))}{v^{p}(\tilde{x})} p v^{p-1}(\tilde{x})
$$

and

$$
c_{2}(x)=-\frac{g(u(\bar{x}))}{u^{q}(\bar{x})} q u^{q-1}(\bar{x}) .
$$


By assumption (b), we know that for $|\tilde{x}|,|\bar{x}|$ large,

$$
\begin{aligned}
& c_{1}(\tilde{x}) \sim \frac{1}{|\tilde{x}|^{b(p-1)}} \sim o\left(\frac{1}{|\tilde{x}|^{\alpha}}\right), \\
& c_{2}(\bar{x}) \sim \frac{1}{|\bar{x}|^{a(q-1)}} \sim o\left(\frac{1}{|\bar{x}|^{\beta}}\right) .
\end{aligned}
$$

By Lemma 1 (decay at infinity), for $\lambda$ sufficiently negative (less than the $R$ in Lemma 1), it holds that

$$
\tilde{U}_{\lambda}(x), \tilde{V}_{\lambda}(x) \geq 0, \quad x \in \Sigma_{\lambda} .
$$

This completes step 1.

Step 2. Continue to move the plane $T_{\lambda}$ until the limiting position

$$
\lambda_{o}=\sup \left\{\lambda<\infty \mid U_{\rho}(x), V_{\rho}(x) \geq 0, x \in \Sigma_{\rho}, \forall \rho \leq \lambda\right\}
$$

Obviously,

$$
\lambda_{o}<\infty
$$

Otherwise, for any $\lambda>0$,

$$
u\left(0^{\lambda}\right)>u(0) \geq 0
$$

Meanwhile,

$$
u\left(0^{\lambda}\right) \sim \frac{1}{\left|0^{\lambda}\right|^{n-\alpha}} \rightarrow 0, \quad \lambda \rightarrow \infty .
$$

This is a contradiction.

Similarly, one can move the plane $T_{\lambda}$ from $+\infty$ to the left and show that

$$
U_{\lambda_{o}}(x), V_{\lambda_{o}}(x) \leq 0, x \in \Sigma_{\lambda_{o}}
$$

Thus,

$$
U_{\lambda_{o}}(x), V_{\lambda_{o}}(x) \equiv 0, x \in \Sigma_{\lambda_{o}} .
$$

Due to the arbitrariness of the $x_{1}$ direction, we conclude that $u, v$ are symmetric about some point in $R^{n}$. 


\section{Proof of Theorem 3}

Without any decay assumption on $u$ and $v$, we first consider their Kelvin transform. For any $x^{o} \in R^{n}$, let $\bar{u}$ and $\bar{v}$ be the Kelvin transform of $u$ and $v$ respectively:

$$
\begin{aligned}
& \bar{u}=\frac{1}{\left|x-x^{o}\right|^{n-\alpha}} u\left(\frac{x-x^{o}}{\left|x-x^{o}\right|^{2}}+x^{o}\right), \\
& \bar{v}=\frac{1}{\left|x-x^{o}\right|^{n-\beta}} v\left(\frac{x-x^{o}}{\left|x-x^{o}\right|^{2}}+x^{o}\right) .
\end{aligned}
$$

Without loss of generality, let $x^{o}=0$, then

$$
\begin{aligned}
& \bar{u}=\frac{1}{|x|^{n-\alpha}} u\left(\frac{x}{|x|^{2}}\right), \\
& \bar{v}=\frac{1}{|x|^{n-\beta}} v\left(\frac{x}{|x|^{2}}\right) .
\end{aligned}
$$

By (7) and (29),

$$
\begin{aligned}
(-\triangle)^{\alpha / 2} \bar{u}(x) & =\frac{1}{|x|^{n+\alpha}}\left[(-\triangle)^{\alpha / 2} u\right]\left(\frac{x}{|x|^{2}}\right) \\
& =\frac{1}{|x|^{n+\alpha}} f\left(|x|^{n-\beta} \bar{v}(x)\right) .
\end{aligned}
$$

Similarly,

$$
(-\triangle)^{\beta / 2} \bar{v}(x)=\frac{1}{|x|^{n+\beta}} g\left(|x|^{n-\alpha} \bar{u}(x)\right)
$$

Let

$$
U_{\lambda}(x)=\bar{u}_{\lambda}(x)-\bar{u}(x), \quad V_{\lambda}(x)=\bar{v}_{\lambda}(x)-\bar{v}(x) .
$$

Then

$$
\begin{aligned}
& (-\triangle)^{\alpha / 2} U_{\lambda}(x)=\frac{f\left(\left|x^{\lambda}\right|^{n-\beta} \bar{v}_{\lambda}(x)\right)}{\left|x^{\lambda}\right|^{n+\alpha}}-\frac{f\left(|x|^{n-\beta} \bar{v}(x)\right)}{|x|^{n+\alpha}}, \\
& (-\triangle)^{\beta / 2} V_{\lambda}(x)=\frac{g\left(\left|x^{\lambda}\right|^{n-\alpha} \bar{u}_{\lambda}(x)\right)}{\left|x^{\lambda}\right|^{n+\beta}}-\frac{g\left(|x|^{n-\alpha} \bar{u}(x)\right)}{|x|^{n+\beta}} .
\end{aligned}
$$

Step 1. Start moving the plane $T_{\lambda}$ from $-\infty$ to the right along the $x_{1}$-axis. 
We will show that for $\lambda$ sufficiently negative,

$$
U_{\lambda}(x), V_{\lambda}(x) \geq 0, x \in \Sigma_{\lambda} \backslash\left\{0^{\lambda}\right\} .
$$

We noticed that for $\lambda$ sufficiently negative, there exists a positive constant $C$ such that

$$
U_{\lambda}(x), V_{\lambda}(x) \geq C>0, x \in B_{\varepsilon}\left(0^{\lambda}\right) \backslash\left\{0^{\lambda}\right\} .
$$

The proof will be given in the appendix.

Meanwhile, by definition, for $\lambda$ fixed,

$$
U_{\lambda}(x), V_{\lambda}(x) \rightarrow 0, \text { as }|x| \rightarrow \infty .
$$

Hence if

$$
\Sigma_{U_{\lambda}}^{-}=\left\{x \in \Sigma_{\lambda} \mid U_{\lambda}(x)<0\right\} \neq \emptyset,
$$

there must exist a point $\tilde{x}$ such that

$$
U_{\lambda}(\tilde{x})=\min _{\Sigma_{\lambda}} U_{\lambda}<0 .
$$

Through arguments similar to those in Lemma 1, we obtain

$$
(-\triangle)^{\alpha / 2} U_{\lambda}(\tilde{x}) \leq \frac{U_{\lambda}(\tilde{x})}{|\tilde{x}|^{\alpha}}<0 .
$$

We claim that

$$
V_{\lambda}(\tilde{x})<0 .
$$

Indeed, otherwise, we have $V_{\lambda}(\tilde{x}) \geq 0$. From (32),

$$
\begin{aligned}
& (-\triangle)^{\alpha / 2} U_{\lambda}(\tilde{x}) \\
= & \frac{f\left(\left|\tilde{x}^{\lambda}\right|^{n-\beta} \bar{v}_{\lambda}(\tilde{x})\right)}{\left|\tilde{x}^{\lambda}\right|^{n+\alpha}}-\frac{f\left(|\tilde{x}|^{n-\beta} \bar{v}(\tilde{x})\right)}{|\tilde{x}|^{n+\alpha}} \\
= & \frac{f\left(\left|\tilde{x}^{\lambda}\right|^{n-\beta} \bar{v}_{\lambda}(\tilde{x})\right)}{\left[\left|\tilde{x}^{\lambda}\right|^{n-\beta} \bar{v}_{\lambda}(\tilde{x})\right]^{p}} \bar{v}_{\lambda}^{p}(\tilde{x})-\frac{f\left(|\tilde{x}|^{n-\beta} \bar{v}_{\lambda}(\tilde{x})\right)}{\left[|\tilde{x}|^{n-\beta} \bar{v}_{\lambda}(\tilde{x})\right]^{p}} \bar{v}_{\lambda}^{p}(\tilde{x}) \\
& +\frac{f\left(|\tilde{x}|^{n-\beta} \bar{v}_{\lambda}(\tilde{x})\right)}{|\tilde{x}|^{n+\alpha}}-\frac{f\left(|\tilde{x}|^{n-\beta} \bar{v}(\tilde{x})\right)}{|\tilde{x}|^{n+\alpha}} \\
\geq & 0 .
\end{aligned}
$$

This is a contradiction with (33), and it proves (34). 
From (34), we can see that there exists a point $\bar{x}$ such that

$$
V_{\lambda}(\bar{x})=\min _{\Sigma_{\lambda}} V_{\lambda}(x)<0,
$$

and

$$
\begin{aligned}
(-\triangle)^{\alpha / 2} U_{\lambda}(\tilde{x}) & =\frac{f\left(\left|\tilde{x}^{\lambda}\right|^{n-\beta} \bar{v}_{\lambda}(\tilde{x})\right)}{\left|\tilde{x}^{\lambda}\right|^{n+\alpha}}-\frac{f\left(|\tilde{x}|^{n-\beta} \bar{v}(\tilde{x})\right)}{|\tilde{x}|^{n+\alpha}} \\
& =\frac{f\left(\left|\tilde{x}^{\lambda}\right|^{n-\beta} \bar{v}_{\lambda}(\tilde{x})\right)}{\left[\left|\tilde{x}^{\lambda}\right|^{n-\beta} \bar{v}_{\lambda}(\tilde{x})\right]^{p}} \bar{v}_{\lambda}^{p}(\tilde{x})-\frac{f\left(|\tilde{x}|^{n-\beta} \bar{v}(\tilde{x})\right)}{\left[\left|\tilde{x}^{\lambda}\right|^{n-\beta} \bar{v}(\tilde{x})\right]^{p}} \bar{v}^{p}(\tilde{x}) \\
& \geq \frac{f\left(|\tilde{x}|^{n-\beta} \bar{v}(\tilde{x})\right)}{\left[\left|\tilde{x}^{\lambda}\right|^{n-\beta} \bar{v}(\tilde{x}) p^{p}\right.}\left[\bar{v}_{\lambda}^{p}(\tilde{x})-\bar{v}^{p}(\tilde{x})\right] \\
& =\frac{f\left(|\tilde{x}|^{n-\beta} \bar{v}(\tilde{x})\right)}{\left[\left|\tilde{x}^{\lambda}\right|^{n-\beta} \bar{v}(\tilde{x})\right]^{p}} p \xi^{p-1} V_{\lambda}(\tilde{x}), \quad \xi \in\left[\bar{v}_{\lambda}^{p}(\tilde{x}), \bar{v}^{p}(\tilde{x})\right] \\
& \geq \frac{f\left(|\tilde{x}|^{n-\beta} \bar{v}(\tilde{x})\right)}{\left[\left|\tilde{x}^{\lambda}\right|^{n-\beta} \bar{v}(\tilde{x})\right]^{p}} p \bar{v}^{p-1} V_{\lambda}(\tilde{x}) \\
& =\frac{p f\left(v\left(\frac{\tilde{x}}{\left.|\tilde{x}|^{2}\right)}\right)\right.}{|\tilde{x}|^{\alpha+\beta} v\left(\frac{\tilde{x}}{|\tilde{x}|^{2}}\right)} V_{\lambda}(\tilde{x}) .
\end{aligned}
$$

Similar to (34), we can show that

$$
U_{\lambda}(\bar{x})<0 .
$$

Then we have

$$
\begin{aligned}
(-\triangle)^{\beta / 2} V_{\lambda}(\bar{x}) & =\frac{g\left(\left|\bar{x}^{\lambda}\right|^{n-\alpha} \bar{u}_{\lambda}(\bar{x})\right)}{\left|\bar{x}^{\lambda}\right|^{n+\beta}}-\frac{g\left(|\bar{x}|^{n-\alpha} \bar{u}(\bar{x})\right)}{|x|^{n+\beta}} \\
& \geq \frac{q g\left(u\left(\frac{\bar{x}}{|\bar{x}|^{2}}\right)\right)}{|\bar{x}|^{\alpha+\beta} u\left(\frac{\bar{x}}{|\bar{x}|^{2}}\right)} U_{\lambda}(\bar{x}) .
\end{aligned}
$$

Let

$$
c_{1}(x)=-\frac{p f\left(v\left(\frac{\tilde{x}}{|\tilde{x}|^{2}}\right)\right)}{|\tilde{x}|^{\alpha+\beta} v\left(\frac{\tilde{x}}{|\tilde{x}|^{2}}\right)},
$$

and

$$
c_{2}(x)=-\frac{q g\left(u\left(\frac{\bar{x}}{|\bar{x}|^{2}}\right)\right)}{|\bar{x}|^{\alpha+\beta} u\left(\frac{\bar{x}}{|\bar{x}|^{2}}\right)} .
$$


From Lemma 1 (decay at infinity), it's easy to deduce that for $\lambda$ sufficiently negative,

$$
U_{\lambda}(x), V_{\lambda}(x) \geq 0, \quad x \in \Sigma_{\lambda} \backslash\left\{0^{\lambda}\right\} .
$$

Step 2. Keeping moving the plane $T_{\lambda}$ until the limiting position

$$
\lambda_{o}=\sup \left\{\lambda \leq 0 \mid U_{\rho}(x), V_{\rho}(x) \geq 0, x \in \Sigma_{\rho} \backslash\left\{0^{\rho}\right\}, \forall \rho \leq \lambda\right\} .
$$

By definition,

$$
U_{\lambda_{o}}(x), V_{\lambda_{o}}(x) \geq 0, \quad x \in \Sigma_{\lambda_{o}} \backslash\left\{0^{\lambda_{o}}\right\} .
$$

There are two possible cases:

i. either

$$
U_{\lambda_{o}}(x)=V_{\lambda_{o}}(x) \equiv 0, \quad x \in \Sigma_{\lambda_{o}} \backslash\left\{0^{\lambda_{o}}\right\},
$$

ii. or

$$
U_{\lambda_{o}}(x), V_{\lambda_{o}}(x)>0, \quad x \in \Sigma_{\lambda_{o}} \backslash\left\{0^{\lambda_{o}}\right\} .
$$

Case $i$. Suppose there exists some $\tilde{x} \in \Sigma_{\lambda_{o}}$ such that

$$
U_{\lambda_{o}}(\tilde{x})=\min _{\Sigma_{\lambda_{o}}} U_{\lambda_{o}}=0
$$

then it must be true that

$$
U_{\lambda_{o}}(x) \equiv 0, \quad x \in \Sigma_{\lambda_{o}} .
$$

Otherwise,

$$
\begin{aligned}
(-\triangle)^{\alpha / 2} U_{\lambda_{o}}(\tilde{x}) & =C_{n, \alpha} P V \int_{\mathbb{R}^{n}} \frac{-U_{\lambda_{o}}(y)}{\left|x^{0}-y\right|^{n+\alpha}} d y \\
& <0 .
\end{aligned}
$$

On the other hand,

$$
\begin{aligned}
& (-\triangle)^{\alpha / 2} U_{\lambda}(\tilde{x}) \\
= & \frac{f\left(\left|\tilde{x}^{\lambda}\right|^{n-\beta} \bar{v}_{\lambda}(\tilde{x})\right)}{\left|\tilde{x}^{\lambda}\right|^{n+\alpha}}-\frac{f\left(|\tilde{x}|^{n-\beta} \bar{v}(\tilde{x})\right)}{|\tilde{x}|^{n+\alpha}} \\
= & \frac{f\left(\left|\tilde{x}^{\lambda}\right|^{n-\beta} \bar{v}_{\lambda}(\tilde{x})\right)}{\left[\left|\tilde{x}^{\lambda}\right|^{n-\beta} \bar{v}_{\lambda}(\tilde{x})\right]^{p}} \bar{v}_{\lambda}^{p}(\tilde{x})-\frac{f\left(|\tilde{x}|^{n-\beta} \bar{v}_{\lambda}(\tilde{x})\right)}{\left[|\tilde{x}|^{n-\beta} \bar{v}_{\lambda}(\tilde{x})\right]^{p}} \bar{v}_{\lambda}^{p}(\tilde{x}) \\
& +\frac{f\left(|\tilde{x}|^{n-\beta} \bar{v}_{\lambda}(\tilde{x})\right)}{|\tilde{x}|^{n+\alpha}}-\frac{f\left(|\tilde{x}|^{n-\beta} \bar{v}(\tilde{x})\right)}{|\tilde{x}|^{n+\alpha}} \\
\geq & 0 .
\end{aligned}
$$


A contradiction with (39). This proves (38).

Since

$$
U_{\lambda_{o}}(x)=-U_{\lambda_{o}}\left(x^{\lambda_{o}}\right),
$$

we have

$$
U_{\lambda_{o}}(x) \equiv 0, \quad x \in R^{n}
$$

Hence

$$
(-\triangle)^{\alpha / 2} U_{\lambda_{o}}(x)=0 .
$$

Together with (35), one can deduce that

$$
\bar{v}_{\lambda_{o}}(x) \leq \bar{v}(x), \quad x \in \Sigma_{\lambda} .
$$

By the definition of $\lambda_{o}$,

$$
\bar{v}_{\lambda_{o}}(x) \geq \bar{v}(x), \quad x \in \Sigma_{\lambda_{o}} .
$$

Thus

$$
V_{\lambda_{o}}(x)=\bar{v}_{\lambda_{o}}(x)-\bar{v}(x) \equiv 0, \quad x \in \Sigma_{\lambda_{o}},
$$

and

$$
V_{\lambda_{o}}(x) \equiv 0, \quad x \in R^{n}
$$

Similarly, if $V_{\lambda_{o}}(x)=0$ somewhere, then we can show that

$$
V_{\lambda_{o}}(x)=U_{\lambda_{o}}(x) \equiv 0, \quad x \in R^{n}
$$

Hence for all $x \in R^{n}$,

$$
\begin{aligned}
0 & =(-\triangle)^{\alpha / 2} U_{\lambda_{o}}(x) \\
& =\frac{f\left(\left|x^{\lambda_{o}}\right|^{n-\beta} \bar{v}_{\lambda_{o}}(x)\right)}{\left|x^{\lambda_{o}}\right|^{n+\alpha}}-\frac{f\left(|x|^{n-\beta} \bar{v}(x)\right)}{|x|^{n+\alpha}} \\
& =\frac{f\left(\left|x^{\lambda_{o}}\right|^{n-\beta} \bar{v}_{\lambda_{o}}(x)\right)}{\left(\left|x^{\lambda_{o}}\right|{ }^{n-\beta} \bar{v}_{\lambda_{o}}(x)\right)^{\frac{n+\alpha}{n-\beta}}} \bar{v}_{\lambda_{o}}^{\frac{n+\alpha}{n-\beta}}(x)-\frac{f\left(|x|^{n-\beta} \bar{v}(x)\right)}{\left(|x|^{n-\beta} \bar{v}(x)\right)^{\frac{n+\alpha}{n-\beta}}} \bar{v}^{\frac{n+\alpha}{n-\beta}}(x) \\
& =\bar{v}^{\frac{n+\alpha}{n-\beta}}(x)\left(\frac{f\left(\left|x^{\lambda_{o}}\right|^{n-\beta} \bar{v}_{\lambda_{o}}(x)\right)}{\left(\left|x^{\lambda_{o}}\right|^{n-\beta} \bar{v}_{\lambda_{o}}(x)\right)^{\frac{n+\alpha}{n-\beta}}}-\frac{f\left(|x|^{n-\beta} \bar{v}(x)\right)}{\left(|x|^{n-\beta} \bar{v}(x)\right)^{\frac{n+\alpha}{n-\beta}}}\right) .
\end{aligned}
$$

Therefore,

$$
\frac{f(t)}{t^{\frac{n+\alpha}{n-\beta}}}=C
$$


Similarly, we can prove that

$$
\frac{g(t)}{t^{\frac{n+\beta}{n-\alpha}}}=C
$$

Case ii. If

$$
U_{\lambda_{o}}(x), V_{\lambda_{o}}(x)>0, \quad x \in \Sigma_{\lambda_{o}},
$$

then we claim that

$$
\lambda_{o}=0 .
$$

To prove (42), suppose that $\lambda_{o}<0$. Then with (41), we would be able to keep moving the plane $T_{\lambda}$. To be precise, for some $\varepsilon>0$ small such $\lambda_{o}+\varepsilon<0$, it holds that

$$
U_{\lambda}(x), V_{\lambda}(x) \geq 0, \quad x \in \Sigma_{\lambda} \backslash\left\{0^{\lambda}\right\}, \forall \lambda \in\left(\lambda_{o}, \lambda_{o}+\varepsilon\right) .
$$

This is a contradiction with the definition of $\lambda_{o}$. Therefore,

$$
\lambda_{o}=0 .
$$

From (41), we have

$$
U_{\lambda_{o}}(x)>0, \quad x \in\left(\Sigma_{\lambda_{o}} \backslash\left\{0^{\lambda_{o}}\right\}\right) \cap B_{R}(0) .
$$

Later, in Lemma 6.2, we will show that

$$
U_{\lambda_{o}}(x) \geq C>0, \quad x \in B_{\varepsilon}\left(0^{\lambda_{o}}\right) \backslash\left\{0^{\lambda_{o}}\right\} .
$$

Together with bounded-away-from- 0 results, we derive that for $\delta>0$ small there exists some $C$ that

$$
U_{\lambda_{o}}(x) \geq C>0, \quad x \in\left(\Sigma_{\lambda_{o}-\delta} \backslash\left\{0^{\lambda_{o}}\right\}\right) \cap B_{R}(0) .
$$

For $\delta, \varepsilon \ll\left|\lambda_{o}\right|$,

$$
0^{\lambda} \in\left(\Sigma_{\lambda_{o}-\delta} \backslash\left\{0^{\lambda_{o}}\right\}\right) \cap B_{R}(0) .
$$

Since $U_{\lambda}(x)$ depends on $\lambda$ continuously,

$$
U_{\lambda}(x) \geq 0, \quad x \in\left(\Sigma_{\lambda_{o}-\delta} \backslash\left\{0^{\lambda_{o}}\right\}\right) \cap B_{R}(0) .
$$

What remains is to show that

$$
U_{\lambda}(x) \geq 0, \quad x \in\left(\Sigma_{\lambda} \backslash \Sigma_{\lambda_{o}-\delta}\right) \cap B_{R}(0) .
$$


We argue by contradiction. Suppose (45) does not holds, then there exists some $\tilde{x} \in\left(\Sigma_{\lambda} \backslash \Sigma_{\lambda_{o}-\delta}\right) \cap B_{R}(0)$ such that

$$
U_{\lambda}(\tilde{x})=\min _{\Sigma_{\lambda}} U_{\lambda}<0 .
$$

Similar to (34), we can show that

$$
V_{\lambda}(\tilde{x})<0
$$

Therefore, there exists some $\bar{x} \in\left(\Sigma_{\lambda} \backslash \Sigma_{\lambda_{o}}\right) \cap B_{R}(0)$ such that

$$
V_{\lambda}(\bar{x})=\min _{\Sigma_{\lambda}} V_{\lambda}<0
$$

By (18),

$$
(-\triangle)^{\alpha / 2} U_{\lambda}(\tilde{x}) \leq \frac{U_{\lambda}(\tilde{x})}{(\delta+\varepsilon)^{\alpha}}<0 .
$$

Similarly, one can show that

$$
(-\triangle)^{\beta / 2} V_{\lambda}(\bar{x}) \leq \frac{V_{\lambda}(\bar{x})}{(\delta+\varepsilon)^{\beta}}<0 .
$$

Since

$$
\tilde{x}, \bar{x} \in\left(\Sigma_{\lambda} \backslash \Sigma_{\lambda_{o}-\delta}\right) \cap B_{R}(0),
$$

we can choose $\delta, \varepsilon \ll \frac{\lambda_{o}}{2}$ such that $|\tilde{x}|,|\bar{x}|>\frac{2 \lambda_{o}}{3}$. Then for some $C$, it holds that

$$
v\left(\frac{\tilde{x}}{|\tilde{x}|^{2}}\right), u\left(\frac{\bar{x}}{|\bar{x}|^{2}}\right) \leq C
$$

and

$$
\frac{1}{(\delta+\varepsilon)^{\alpha+\beta}}>C+2>c_{1}(\tilde{x}) c_{2}(\bar{x}) .
$$

It then follows from Lemma 2 (narrow region principle) that (45) must be true.

Combining (44) and (45), we arrive at

$$
U_{\lambda}(x) \geq 0, \quad x \in \Sigma_{\lambda} \backslash\left\{0^{\lambda}\right\} .
$$

The proof for $V_{\lambda}(x)$ is almost the same and we omit it. This completes the proof of (43) and (42). 
Through an identical argument, one can move $T_{\lambda}$ from $+\infty$ to the left and show that

$$
U_{\lambda_{o}}(x), V_{\lambda_{o}}(x) \leq 0, \quad x \in \Sigma_{\lambda_{o}} \backslash\left\{0^{\lambda_{o}}\right\},
$$

with

$$
\lambda_{o}=0 .
$$

Together with (37), we obtain

$$
U_{0}(x), V_{0}(x) \equiv 0, \quad x \in \Sigma_{0} .
$$

For a more general Kelvin transform as in (27) and (28), through a similar argument as above one can show that

$$
\lambda_{o}=x_{1}^{o},
$$

and

$$
U_{\lambda_{o}}(x), V_{\lambda_{o}}(x) \equiv 0, \quad x \in \Sigma_{\lambda_{o}} .
$$

For any $x^{1}, x^{2} \in R^{n}$, let their midpoint be the center of the Kelvin transform:

$$
x^{o}=\frac{x^{1}+x^{2}}{2} .
$$

Let

$$
y^{1}=\frac{x^{1}-x^{o}}{\left|x^{1}-x^{o}\right|^{2}}+x^{o}, \quad y^{2}=\frac{x^{2}-x^{o}}{\left|x^{2}-x^{o}\right|^{2}}+x^{o} .
$$

Then

$$
y^{2}=\left(y^{1}\right)^{\lambda_{o}},
$$

and

$$
\bar{u}\left(y^{1}\right)=\bar{u}\left(y^{2}\right), \quad \bar{v}\left(y^{1}\right)=\bar{v}\left(y^{2}\right) .
$$

It thus implies that

$$
u\left(x^{1}\right)=u\left(x^{2}\right), \quad v\left(x^{1}\right)=v\left(x^{2}\right) .
$$

Since $x^{1}, x^{2}$ are arbitrary, $u$ and $v$ must be constant.

Combining Case $i$ and Case ii, we complete the proof. 


\section{Proof of Theorem 4}

From Theorem [3, we know that when $\alpha=\beta$, if $u$ and $v$ are nonnegative solutions for (17), then

- either $u$ and $v$ are constant,

- or $f(v)=C_{1} v^{\frac{n+\alpha}{n-\alpha}}$ and $g(u)=C_{2} u^{\frac{n+\alpha}{n-\alpha}}$.

By Theorem 2 in [24], we know that when $\alpha=\beta$, system (7) is equivalent to

$$
\left\{\begin{array}{l}
u(x)=\int_{R^{n}} \frac{C_{1} v^{\frac{n+\alpha}{n-\alpha}}(y)}{|x-y|^{n-\alpha}} d y \\
v(x)=\int_{R^{n}} \frac{C_{2} u^{\frac{n+\alpha}{n-\alpha}}(y)}{|x-y|^{n-\alpha}} d y
\end{array}\right.
$$

From the results in [35], we have

$$
u(x)=C_{1}\left(\frac{c}{c^{2}+\left|x-x_{0}\right|^{2}}\right)^{\frac{n-\alpha}{2}}, \quad v(x)=C_{2}\left(\frac{c}{c^{2}+\left|x-x_{0}\right|^{2}}\right)^{\frac{n-\alpha}{2}} .
$$

This proves the theorem.

\section{Appendix}

We use the ideas in the proof of the bounded-away-from-0 lemmas in the appendix in [31].

Lemma 6.1 For $\lambda$ sufficiently negative, there exists a positive constant $C$ such that

$$
U_{\lambda}(x), V_{\lambda}(x) \geq C>0, x \in B_{\varepsilon}\left(0^{\lambda}\right) \backslash\left\{0^{\lambda}\right\}
$$

Proof. For $\lambda \in \Sigma_{\lambda}$, as $\lambda \rightarrow-\infty$, it's easy to see that

$$
\bar{u}(x) \rightarrow 0 \text {. }
$$

To prove (46), it suffices to show that

$$
\bar{u}_{\lambda}(x) \geq C>0, \quad x \in B_{\varepsilon}\left(0^{\lambda}\right) \backslash\left\{0^{\lambda}\right\} .
$$

Or equivalently,

$$
\bar{u}(x) \geq C>0, \quad x \in B_{\varepsilon}(0) \backslash\{0\} .
$$


Let $\eta$ be a smooth cutoff function such that $\eta(x) \in[0,1]$ in $\mathbb{R}^{n}$, supp $\eta \subset$ $B_{2}$ and $\eta(x) \equiv 1$ in $B_{1}$. Let

$$
(-\triangle)^{\alpha / 2} \varphi(x)=\eta(x) f(v)(x) .
$$

Then

$$
\varphi(x)=C_{n,-\alpha} \int_{\mathbb{R}^{n}} \frac{\eta(y) f(v)(y)}{|x-y|^{n-\alpha}} d y=C_{n,-\alpha} \int_{B_{2}(0)} \frac{\eta(y) f(v)(y)}{|x-y|^{n-\alpha}} d y .
$$

It's trivial that for $|x|$ sufficiently large,

$$
\varphi(x) \sim \frac{1}{|x|^{n-\alpha}} .
$$

Since

$$
\begin{cases}(-\triangle)^{\alpha / 2}(u-\varphi) \geq 0, & x \in B_{R}, \\ (u-\varphi)(x) \geq 0, & x \in B_{R}^{c},\end{cases}
$$

by the maximum principle (see [36]), we have

$$
(u-\varphi)(x) \geq 0, \quad x \in B_{R} .
$$

Thus

$$
(u-\varphi)(x) \geq 0, \quad x \in R^{n} .
$$

For $|x|$ sufficiently large, from (48), one can see that for some constant $C>0$,

$$
u(x) \geq \frac{C}{|x|^{n-\alpha}} .
$$

Hence for $|x|$ small

$$
u\left(\frac{x}{|x|^{2}}\right) \geq C|x|^{n-\alpha},
$$

and

$$
\bar{u}(x)=\frac{1}{|x|^{n-\alpha}} u\left(\frac{x}{|x|^{2}}\right) \geq C .
$$

Together with (47), it yields that

$$
U_{\lambda}(x) \geq \frac{C}{2}>0, \quad x \in B_{\varepsilon}\left(0^{\lambda}\right) \backslash\left\{0^{\lambda}\right\} .
$$

Through an identical argument, one can show that (51) holds for $V_{\lambda}(x)$ as well.

This completes the proof of the lemma. 
Lemma 6.2 For $\lambda_{o}<0$, if either of $U_{\lambda_{o}}(x), V_{\lambda_{o}}(x)$ is not identically 0 , then there exists some constant $C$ and $\varepsilon>0$ small such that

$$
U_{\lambda_{o}}(x), V_{\lambda_{o}}(x) \geq C>0, \quad x \in B_{\varepsilon}\left(0^{\lambda_{o}}\right) \backslash\left\{0^{\lambda_{o}}\right\} .
$$

Proof. From Lemma 2.2 in [23], we have the integral expression of $U_{\lambda_{o}}$ :

$$
\begin{aligned}
U_{\lambda_{o}}(x)= & C_{n, \alpha} \int_{\Sigma_{\lambda_{o}}}\left(\frac{1}{|x-y|^{n-\alpha}}-\frac{1}{\left|x-y^{\lambda_{o}}\right|^{n-\alpha}}\right) \\
& \cdot\left(\frac{f\left(\left.y^{\lambda_{o}}\right|^{n-\beta} \bar{v}_{\lambda_{o}}(y)\right)}{\left|y^{\lambda_{o}}\right|^{n+\alpha}}-\frac{f\left(|y|^{n-\beta} \bar{v}(y)\right)}{|y|^{n+\alpha}}\right) d y \\
= & C_{n, \alpha} \int_{\Sigma_{\lambda_{o}}}\left(\frac{1}{|x-y|^{n-\alpha}}-\frac{1}{\left|x-y^{\lambda_{o}}\right|^{n-\alpha}}\right) \\
& \cdot\left(\frac{f\left(\left|y^{\lambda_{o}}\right|^{n-\beta} \bar{v}_{\lambda_{o}}(y)\right)}{\left[\left|y^{\lambda_{o}}\right|^{n-\beta} \bar{v}_{\lambda_{o}}(y)\right]^{p}} \bar{v}_{\lambda_{o}}^{p}(y)-\frac{f\left(|y|^{n-\beta} \bar{v}_{\lambda_{o}}(y)\right)}{\left[|y|^{n-\beta} \bar{v}_{\lambda_{o}}(y)\right]^{p}} \bar{v}_{\lambda_{o}}^{p}(y)\right. \\
& \left.+\frac{f\left(|y|^{n-\beta} \bar{v}_{\lambda_{o}}(y)\right)-f\left(|y|^{n-\beta} \bar{v}(y)\right)}{|y|^{n+\alpha}}\right) d y \\
\geq & C_{n, \alpha} \int_{\Sigma_{\lambda_{o}}}\left(\frac{1}{|x-y|^{n-\alpha}}-\frac{1}{\left|x-y^{\lambda_{o}}\right|^{n-\alpha}}\right) \\
& \cdot \frac{f\left(|y|^{n-\beta} \bar{v}_{\lambda_{o}}(y)\right)-f\left(|y|^{n-\beta} \bar{v}(y)\right)}{|y|^{n+\alpha}} d y .
\end{aligned}
$$

Since

$$
V_{\lambda_{0}}(x) \not \equiv 0, \quad x \in \Sigma_{\lambda_{0}}
$$

there exists some $x^{0}$ such that

$$
V_{\lambda_{o}}\left(x^{0}\right)>0
$$

Thus, for some $\delta>0$ small, it holds that

$$
f\left(|y|^{n-\beta} \bar{v}_{\lambda_{o}}(y)\right)-f\left(|y|^{n-\beta} \bar{v}(y)\right) \geq C>0, \quad y \in B_{\delta}\left(x^{0}\right) .
$$

Therefore,

$$
U_{\lambda_{o}}(x) \geq \int_{B_{\delta}\left(x^{0}\right)} C d y \geq C>0 .
$$

In a same way, one can show that $V_{\lambda_{o}}(x)$ also satisfies (52). This completes the proof. 


\section{References}

[1] Ambrosetti A, Rabinowitz P. Dual variational methods in critical points theory and applications. J. Funct. Anal. 1973, 14: 349-381

[2] Gidas B, Spruck J. A priori bounds for positive solutions of nonlinear elliptic equations, Comm. Partial Differential Equations, 1981, 6: 883901

[3] Pohozaev S. I. On the eigenfunctions of the equation $\mathcal{A} u+\lambda f(u)=0$, Dokl. Akad. Nauk SSSR. 1965, 165: 1408-1411

[4] Rabinowitz P.H. Minimax Methods in Critical Point Theory with Applications to Differential Equations, CBMS Reg. Conf. Ser. Math., vol. 65, American Mathematical Society, Providence, RI, 1986

[F] Fiscella A. Saddle point solutions for nonlocal elliptic operators, Topol. Methods Nonlinear Anal. 2014, 44: 527-538

[SeV] Servadei R, Valdinoci E. Mountain pass solutions for non-local elliptic operators, J. Math. Anal. Appl. 2012, 389: 887-898

[5] Pucci P, Radulescu V. The impact of the mountain pass theory in nonlinear analysis: a mathematical survey, Boll. Unione Mat. Ital. 2010, 3: $543-584$

[6] Mitidieri E. A Rellich type identity and applications, Comm. Partial Differential Equations, 1993, 18: 125-151

[7] Serrin J, Zou H. Non-existence of positive solutions of Lane-Emden systems, Differential Integral Equations 1996, 9: 635-653

[8] Poláčcik P, Quittner P, Souplet P. Singularity and decay estimates in superlinear problems via Liouville-type theorems. Part I: Elliptic systems, Duke Math. J. 2007, 139: 555-579

[9] Souplet P. The proof of the Lane-Emden conjecture in four space dimensions, Adv. in Mathematics, 2009, 221: 1409-1427

[10] Figueiredo D.-D, Felmer P. A Liouville-type theorem for elliptic systems, Annali della Scuola Normale Superiore di Pisa-Classe di Scienze, 1994, 21: 387-397 
[11] Chen W, Li C. An integral system and the Lane-Emden conjecture, Disc. \& Cont. Dynamics Sys. 2009, 4: 1167-1184

[12] Busca J, Mansevich R. A Liouville-type theorem for LaneEmden system, Indiana Univ. Math. J. 2002, 51: 37-51.

[13] Lin C.-S. A classification of solutions of a conformally invariant fourth order equation in $R^{n}$, Comment. Math. Helv. 1998, 73: 206-231

[14] Yu X. Liouville type theorems for integral equations and integral systems, Calc. Var. 2013, 46: 75-95

[15] Arthur A, Yan X, Zhao M. A Liouville-type theorem for higher order elliptic systems, Disc. \& Cont. Dynamics Sys. 2014, 34: 3317-3339

[16] Bertoin J. Lévy Processes Cambridge Tracts in Math. vol. 121, Cambridge: Cambridge University Press, 1996

[17] Valdinoci E. From the long jump random walk to the fractional Laplacian, Bol. Soc. Esp. Mat. Apl. SeMA, 2009, 49: 33-44

[18] Caffarelli L, Roquejoffre J.-M, Sire Y. Variational problems for free boundaries for the fractional Laplacian, J. Eur. Math. Soc. (JEMS) 2010, 12: 1151-1179

[19] Cont R, Tankov P. Financial Modelling with Jump Processes, Chapman and Hall/CRC Financ. Math. Ser. Chapman and Hall/CRC, Boca Raton, FL, 2004

[20] Duvaut G, Lions J.-L. Inequalities in Mechanics and Physics, Grundlehren Math. Wiss. vol. 219, Springer-Verlag, Berlin, 1976, translated from French by C.W. John

[SiV] Sire Y, Valdinoci E. Fractional Laplacian phase transitions and boundary reactions: A geometric inequality and a symmetry result J. Funct. Anal. 2009, 256: 1842-1864

[21] Alberti G, Bouchitt G, Seppecher P. Phase transition with the linetension effect. Arch. Ration. Mech. Anal. 1998, 144: 1-46

[22] Gonzlez M.d.M. Gamma convergence of an energy functional related to the fractional Laplacian, Cal. Var. \& PDEs. 2009, 36: 173-210 
[23] Chen W, Fang Y, Yang R. Liouville theorems involving the fractional Laplacian on a half space, Adv. in Mathematics, 2015, 274: 167-198

[24] Zhuo R, Chen W, Cui X, Yuan Z. Symmetry and non-existence of solutions for a nonlinear system involving the fractional Laplacian, Disc. Cont. Dyn. Sys. 2015, 2: 1125-1141

[25] Chen W, Li C. Classification of solutions of some nonlinear elliptic equations, Duke Math. J. 1991, 63: 615-622

[26] Chen W, Li C. Methods on Nonlinear Elliptic Equations, AIMS book series, vol. 4, 2010

[27] Chen W, Li C, Ou B. Classification of solutions for an integral equation, Comm. Pure Appl. Math. 2006, 59: 330-343

[28] Chen W, Li C, Ou B. Qualitative properties of solutions for an integral equation, Disc. Cont. Dyn. Sys. 2005, 12: 347-354

[29] Tang D, Fang Y. Regularity and nonexistence of solutions for a system involving the fractional Laplacian, Comm. Pure Appl. Anal. 2015, 14: $2431-2451$

[30] Leite E, Montenegro M. On positive viscosity solutions of fractional Lane-Emden systems, 2015, arXiv:1509.01267

[31] Chen W, Li C, Li Y. A direct method of moving planes for the fractional Laplacian, 2014, arXiv:1411.1697

[32] Quaas A, Xia A. Liouville type theorems for nonlinear elliptic equations and systems involving fractional Laplacian in the half space, Cal. Var. \& PDEs 2014, 3-4: 641-659

[33] Mou C. Nonlinear elliptic systems involving the fractional Laplacian in the unit ball and on a half space, C.P.A.A. 2015, 6: 2335-2362

[34] Cheng Z, Huang G, Li C. A Liouville theorem for subcritical LaneEmden system, 2014, arXiv:1412.7275

[35] Chen W, Li C, Ou B. Classification of solutions for a system of integral equations. Commun. Partial Differ. Equ. 2005, 30: 59-65 
[36] Silvestre L. Regularity of the obstacle problem for a fractional power of the Laplace operator, Comm. Pure Appl. Math. 2007, 60: 67-112

Authors' Addresses and E-mails:

Yan Li

Department of Mathematical Sciences

Yeshiva University

New York, NY, 10033 USA

yali3@mail.yu.edu

Pei Ma

Jiangsu Key Laboratory for NSLSCS

School of Mathematical Science

Nanjing Normal University

Nanjing, Jiangsu, 210023 China

Department of Mathematical Sciences

Yeshiva University

New York, NY, 10033 USA

mapei0620@126.com 